\title{
«Le problème, ce ne sont pas nos corps, ce sont les barrières!»
}

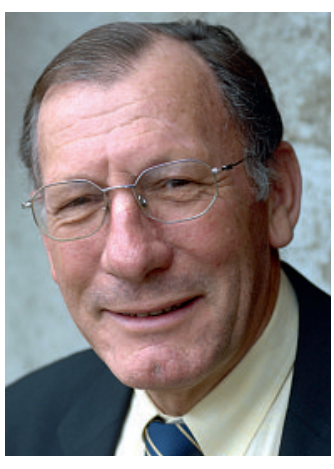

Jean Martin

1 Shakespeare T. Disability, genetics and global justice. Social Policy and Society. 2005; 4(1):87-95

2 Martin J. Méthodes amélioratives (enhancement) et autres avancées. Revue médicale suisse. 2009; 5:606

3 Martin J. L'immortalité? Cauchemar. Bull Méd Suisses. 2009, 90(3): 90.

4 En faisant - là aussi, c'est une remarque de santé publique - une comparaison avec la grossesse à l'adolescence (!): on peut sans doute vivre une telle grossesse de manière heureuse, mais il est néanmoins préférable de ne pas être enceinte trop tôt.

5 L'OMS vient de sortir le premier Rapport mondial sur le handicap (OMS, Genève 2011), indiquant que dans le monde on peut estimer que $15 \%$ de la population présentent un handicap. www.who. int/entity/disabilities/ world_report/2011/fr
Phrase qui étonne... prononcée début juillet, à la Fondation Brocher près de Genève, par Tom Shakespeare, bioéthicien britannique au sein de la section chargée du handicap (disability) au Siège de l'OMS. Lui-même est achondroplasique, est donc limité dans ses aptitudes par sa petite taille et se déplace en chaise roulante (NB: certaines des positions ci-dessous sont remarquables dans la bouche d'une personne handicapée mais pourraient être une expression de banalisation et de manque d'engagement dans celle d'un autre). Il faisait un exposé dans le cadre d'une Académie d'été organisée par le Hastings Center (New York) consacrée à la problématique de $\mathrm{l}^{\prime}$ «amélioration de l'être humain» (enhancement).

Il a eu cette formule en parlant de promotion des droits des handicapés, par l'OMS et d'autres, droits à une vie aussi libérée de contraintes que possible. Elle m'a frappé comme illustrant vivement ce qu'est la santé publique (public health): en effet, la branche de la médecine et de la politique de santé qui a pour objet le bien-être optimal des gens dans la société veut permettre d'avoir la vie la plus autonome, la plus «praticable» et satisfaisante possible, dans son milieu; une vie dans laquelle on ne se voit pas interdire l'accès aux potentialités de réalisation et de plaisir qu'ont la plupart de nos congénères. Shakespeare a écrit «le handicap est le produit de l'interaction entre des corps 〈limités (impaired) et des environnements sociaux qui excluent.» [1]

Par barrières, il faut entendre ici tout ce qui nous bloque, diminue la qualité de vie. Qualité de vie liée, entre autres facteurs, à l'accès à une eau et des aliments sains, à la lutte contre les maladies infectieuses, à l'évitement de pollutions de toutes sortes, à la libération de la dépendance au tabac et d'autres produits, à ce qui rend malaisé l'exercice physique, etc.

Revenant au handicap, on sait les progrès importants réalisés par l'abolition des obstacles architecturaux, qu'il s'agisse d'environnement extérieur ou des bâtiments publics ou privés, et par les moyens (mécaniques, électriques, électroniques) permettant de se déplacer, d'accomplir tant de tâches de la vie quotidienne, d'utiliser ordinateurs et appareils. Jusqu'aux «prothèses» qui peuvent suppléer à des fonctions insuffisantes ou absentes, notamment sensorielles. Remarque de Shakespeare à propos de l'allongement des membres inférieurs par la chirurgie, parfois proposé à des achondroplasiques: "pour que je puisse allumer la lumière, il ne s'agit pas d'abord d'allonger mes jambes mais de placer l'interrupteur plus bas.» $\mathrm{Ou}$, à propos de surdité et des moyens d'aider les sourds - et en faisant référence aux pays défavorisés, cible principale de l'OMS: «Le langage des signes lui ne demande pas qu'on dispose de piles électriques.» Mais il ne s'agit bien entendu pas de refuser les moyens modernes.

L'objectif, s'agissant de santé publique et plus largement de politique sociale, est de permettre aux personnes handicapées un épanouissement aussi substantiel que possible. Remarque importante: à mesure que des possibilités nouvelles (techniques notamment) sont disponibles pour les bien-portants, les pouvoirs publics - et les initiatives privées - ont le devoir de mettre en œuvre sans délais les mesures adéquates pour en donner accès aux handicapés aussi. Sinon, on aggrave ou crée de nouvelles contraintes pour ces derniers.

Shakespeare a fait sourire l'audience en évoquant le bonheur, notion si difficile à juger. Il parlait de philosophes radicaux dont l'espoir affiché est le «enhancement», améliorer l'homme pour en faire un surhomme (transhumanisme), voire à le rendre immortel $[2,3]$. On pense à des noms comme ceux de Julian Savulescu, John Harris, Ray Kurzweil. Notant que ces personnes peuvent être des perfectionnistes obsessionnels, doctrinaires qui sont malheureux des imperfections de l'être humain tel que nous le connaissons, il s'est demandé si «malgré mes handicaps, je ne suis pas plus heureux qu'eux». Tant il est vrai que le bonheur n'est pas d'abord une question physique ou fonctionnelle objective, mais un état d'esprit, lié notamment à une capacité d'adaptation. Disant aussi «one could see the notion of disabled as differently able» (capable, mais différemment).

Phrase quelque peu énigmatique: «On a besoin de limites pour bien fonctionner.» Ce qui me fait penser à cette formule que je crois bonne: «La liberté ne vaut que par les limites qu'on accepte de lui mettre.» Terminant sur un plan plus philosophique, Shakespeare a conclu que les personnes handicapées peuvent sans doute avoir une bonne vie (good life), bien qu'il soit plus simple de ne pas être handicapé $[4,5]$.

Jean Martin*

* Jean Martin est médecin de santé publique, membre de la Commission nationale d'éthique et de la rédaction. 